\title{
Möglichkeiten der nicht operativen Behandlung von Pseudarthrosen
}

Jens Everding, Josef Stolberg-Stolberg, Steffen Roßlenbroich, Michael J. Raschke

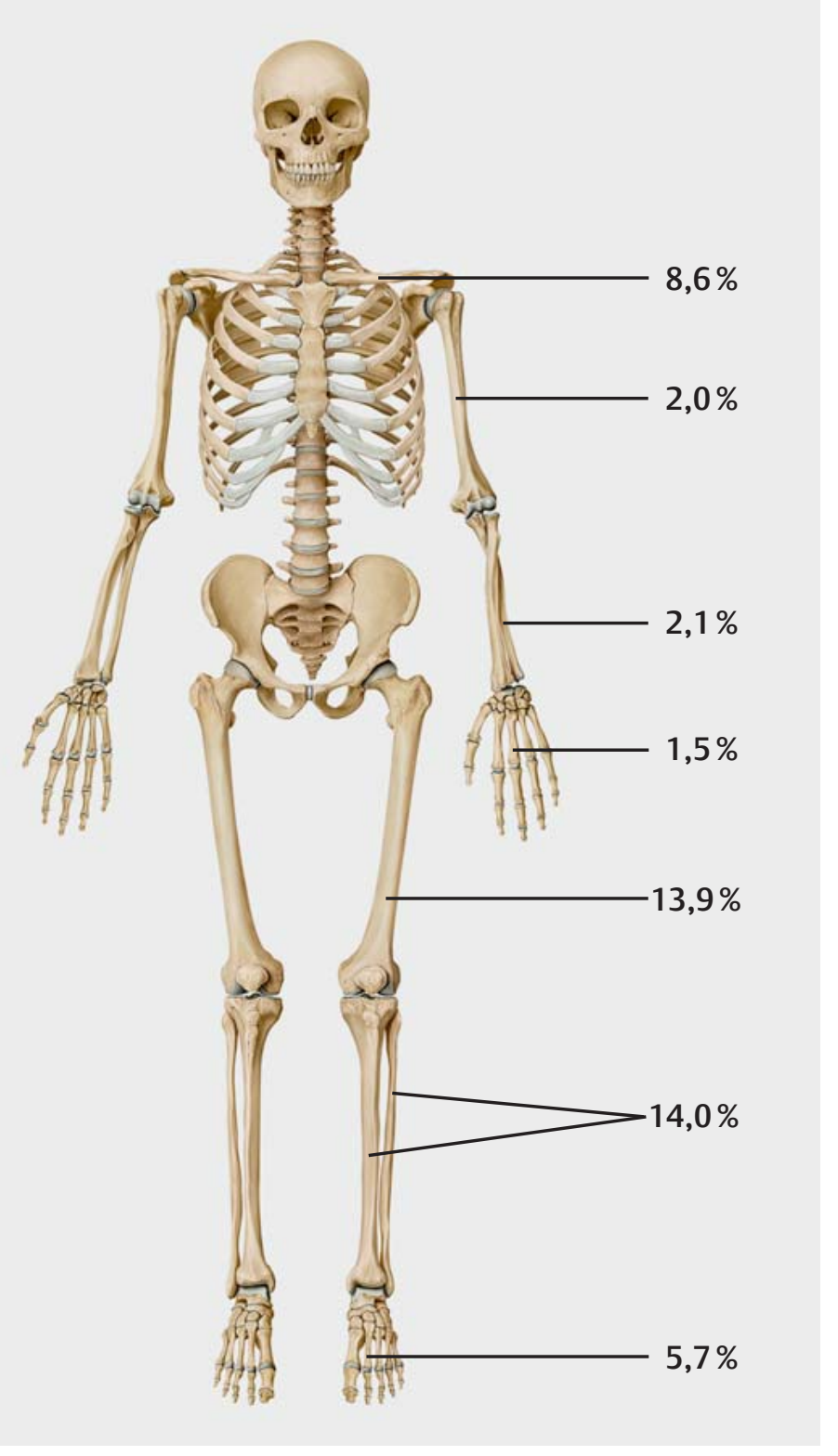

- Abb. 1 Häufigkeit der Pseudarthrosen in Abhängigkeit von der Lokalisation basierend auf den Daten von Zura et al. (2016) [2]. Quelle: Schünke M, Schulte E, Schumacher U et al., Hrsg. Prometheus LernAtlas Allgemeine Anatomie und Bewegungssystem. 5., vollständig überarbeitete Auflage. Thieme; 2018. doi:10.1055/b-006-149643

\section{Epidemiologie}

Pseudarthrosen sind regelmäßig auftretende Verletzungsfolgen. In Deutschland werden jährlich 14000 Patienten mit der Diagnose Nichtvereinigung der Frakturenden (M84.1) stationär behandelt, Männer im Alter von 40-65 Jahren sind besonders häufig betroffen (Statistisches Bundesamt, März 2019). Aufgrund u.a. der hohen mechanischen Belastung sind Pseudarthrosen im überwiegenden Maße an der unteren Extremität lokalisiert. Tzioupis und Giannoudis (2007) konnten in einer Übersichtsarbeit die höchste Prävalenz für tibiale Pseudarthrosen nachweisen, gefolgt von Frakturheilungsstörungen des Femurs. Pseudarthrosen der oberen Extremität sind häufiger an den paarigen Unterarmknochen lokalisiert als am Humerus [1]. In einer weiteren Studie von Zura et al. (2016) wurde bei 309330 Frakturen in 4,9\% die Ausbildung einer Knochenheilungsstörung beobachtet. - Abb. 1 zeigt die Häufigkeiten der Pseudarthrosen in Abhängigkeit von der Lokalisation [2]. Insgesamt variiert die Pseudarthrosenrate in der Literatur stark. Einflussfaktoren wie der begleitende Weichteilschaden und das Therapieverfahren (konservativ vs. Platten- vs. Marknagelosteosynthese), aber auch patientenabhängige Faktoren spielen eine große Rolle [1,3].

\section{Definition und Klassifikation}

Im deutschsprachigen Raum wird eine ausbleibende knöcherne Konsolidierung über einen Zeitraum von 4-6 Monaten als verzögerte Frakturheilung bezeichnet und die fehlende knöcherne Konsolidierung über den 6. Monat hinaus als Pseudarthrose beschrieben. Die European Society of Tissue Regeneration in Orthopedics and Traumatology (ESTROT) und die AO (Arbeitsgemeinschaft für Osteosynthesefragen) definieren die Pseudarthrose als Fraktur, die ohne Intervention, unabhängig von der Behandlungsdauer, nicht verheilen wird. Grundsätzlich beinhaltet die Definition des Begriffs Pseudarthrose einen großen ärztlichen Interpretationsspielraum, weshalb die Diagnosestellung dieser Verletzungsfolge ein großes Maß an Erfahrung auf diesem Gebiet von dem behandelnden Arzt erfordert.

Weber und Czech (1973) klassifizierten Pseudarthrosen in biologisch reaktionsfähig und biologisch inaktiv ( $\triangleright$ Abb. 2) [4]. Als eigenständige Entität kann die Infekt- 


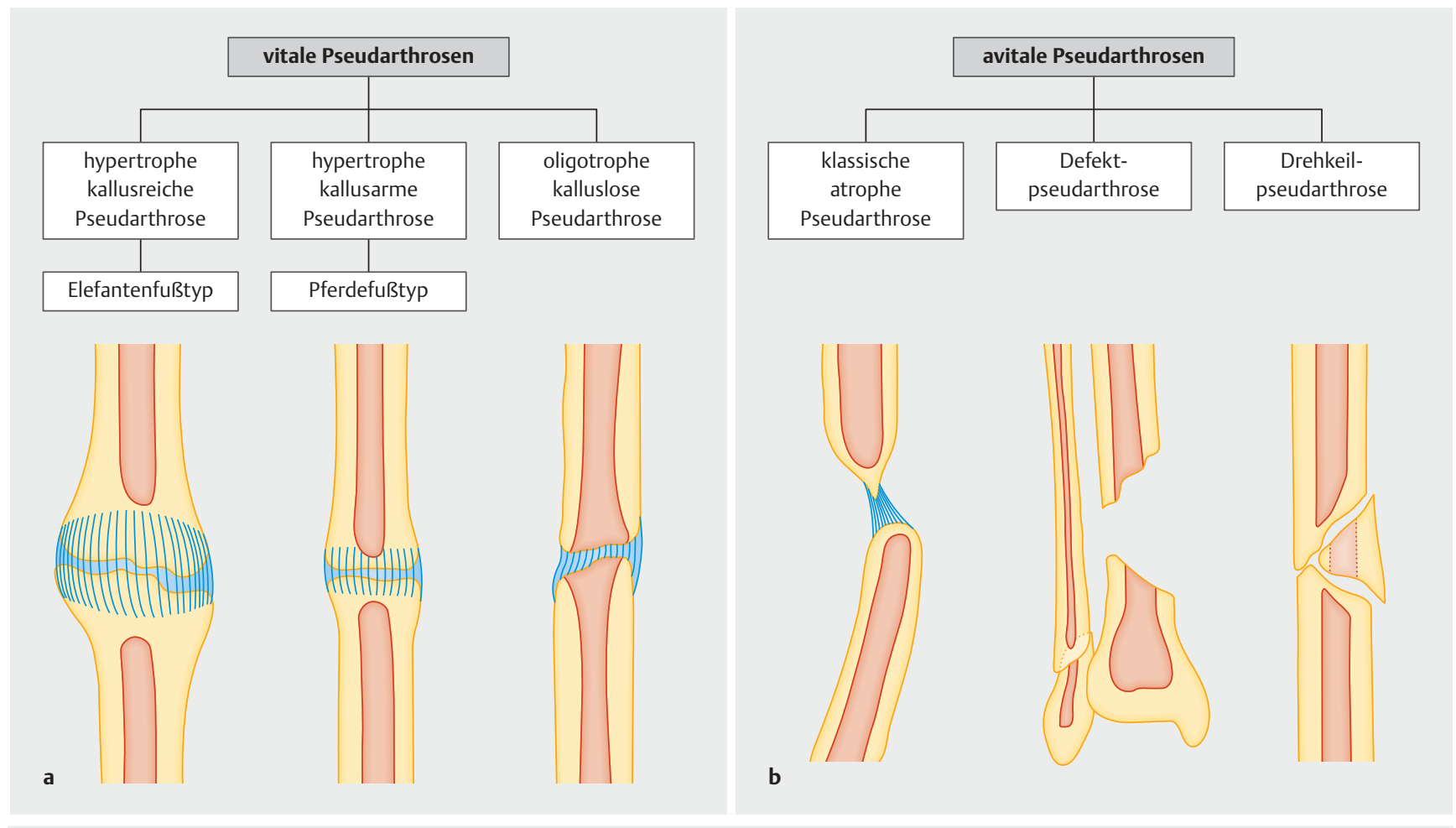

- Abb. 2 Klassifikation der Pseudarthrosen nach Weber und Czech [4]. Quelle: Piatek S. Grundzüge der Knochenbruchheilung und ihrer Störungen. In: Lippert H, Hrsg. Wundatlas. 3. Aufl. Stuttgart: Thieme; 2012. doi:10.1055/b-002-35728.

pseudarthrose und die Defektpseudarthrose angesehen werden. Calori et al. (2008) haben mit dem Non-Union Scoring System (NUSS) ein neues, zwischenzeitlich etabliertes Scoring System entwickelt, das zahlreiche patientenabhängige und patientenunabhängige Faktoren berücksichtigt und die Komplexität der Pseudarthrose widerspiegelt [5].

Eine entscheidende Rolle spielt die bildmorphologische Unterteilung in hypertrophe, atrophe und oligotrophe Pseudarthrosen infolge hieraus resultierender unterschiedlicher Therapieempfehlungen und zugrunde liegender Kausalität. Im Allgemeinen ist die hypertrophe Pseudarthrose durch eine überschießende Kallusreaktion charakterisiert, der eine mangelnde Stabilität zugrunde liegt. Die atrophe Pseudarthrose ist auf eine insuffiziente lokale Biologie zurückzuführen und durch fehlende Kallusbildung in der Bildgebung gekennzeichnet. Oligotrophe Pseudarthrosen stellen ein Mischbild zwischen den beiden erstgenannten Pseudarthrosenarten dar und bedürfen häufig neben einer Verbesserung der mechanischen Stabilität einer Optimierung der lokalen biologischen Verhältnisse.

\section{Indikation zur konservativen Behandlung}

Der Goldstandard in der Behandlung von Pseudarthrosen ist die operative Therapie basierend auf dem Diamond Concept [6]. In bestimmten Fällen ist allerdings eine nicht operative Behandlung von Pseudarthrosen gerechtfertigt. Besonders in der Frühphase können Belastungsaufbau und supportive biophysikalische Verfahren sinnvolle Therapiekonzepte darstellen, um eine erneute Operation abzuwenden. Letzteres umfasst die niedrig intensivierte gepulste Ultraschalltherapie (LIPUS), die hochenergetische fokussierte extrakorporale Stoßwellentherapie (fESWT) und die elektrische bzw. elektromagnetische Therapie (PEMF). Ein großer Vorteil dieser Maßnahmen liegt in der vernachlässigbaren Komplikationsrate. Higgins et al. (2014) beschreiben, dass bei dem Gebrauch von 55000 LIPUS-Geräten (niedrig intensivierte gepulste Ultraschallgeräte) über einen Zeitraum von einem Jahr lediglich 3-mal über Hautirritationen und einmal über Brustschmerzen infolge einer Interaktion mit einem einliegenden Schrittmacher berichtet wurde [7]. Furia et al. (2010) verglichen Konsolidierungsraten von Patienten mit Basisfrakturen des 5. Mittelfußknochens nach fokussierter hochenergetischer extrakorporaler Stoßwellentherapie (fESWT) und Operation: In dieser Studie kam es in Bezug auf die Komplikationsrate in der fESWT-Gruppe 


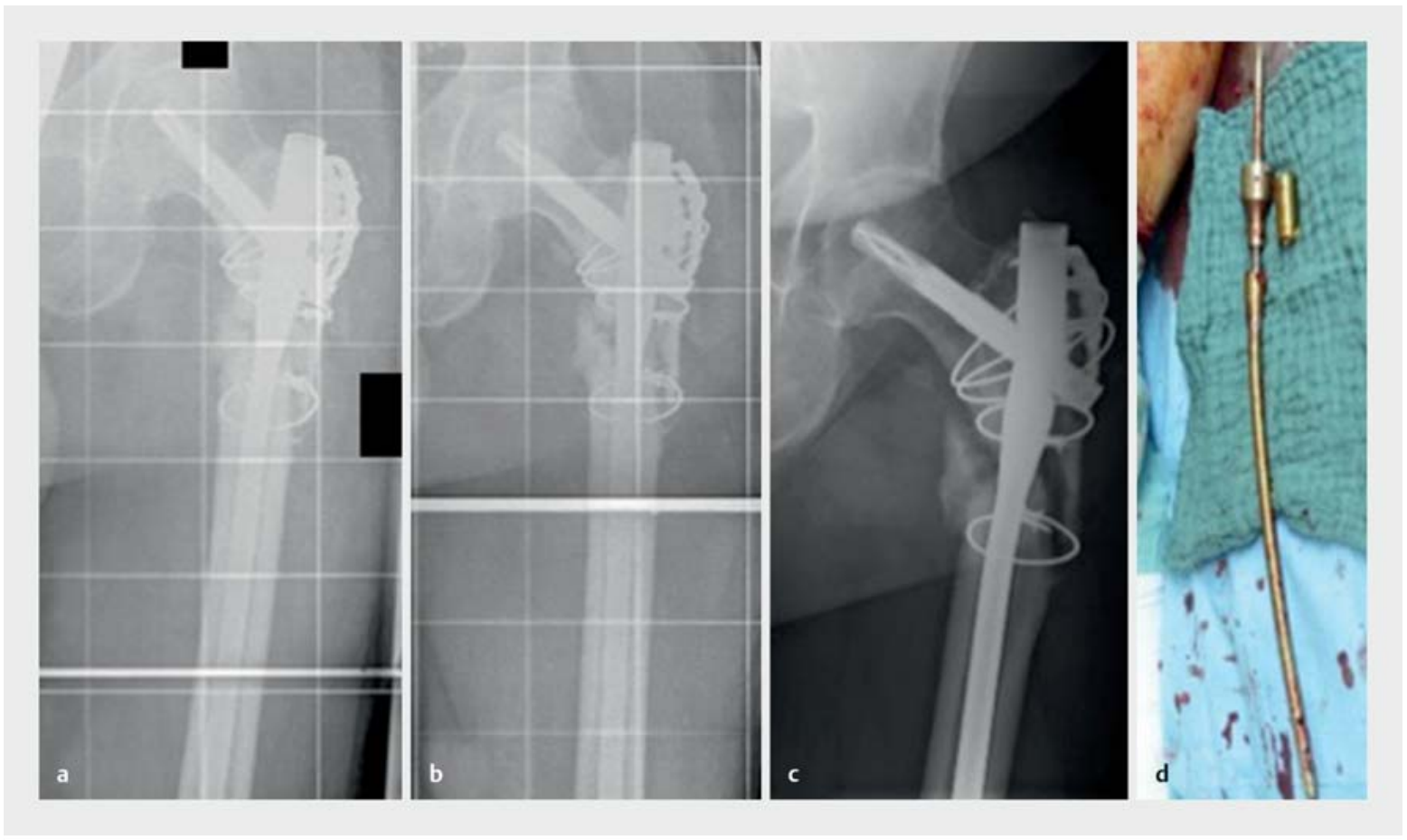

- Abb. 3 Die subtrochantäre Region unterliegt hohen Druck- und Zugkräften bei prädominanter kortikaler Knochenstruktur und geringer Vaskularisierung. In dieser Region sollten biophysikalische Verfahren zurückhaltend angewendet werden. Die Abbildung zeigt einen erfolglosen Versuch der Pseudarthrosenbehandlung mit fESWT: a Pseudarthrose vor fESWT, b 6 Wochen nach fESWT, c, d Implantatversagen mit Bruch des einliegenden Marknagels 3 Monate nach Stoßwellentherapie.

zu einem Hämatom, in der Operationsgruppe traten 11 Komplikationen auf (Refraktur, implantatassoziierte Beschwerden) [8]. Auch in unserem eigenen Patientenkollektiv ergaben sich nach fESWT bis auf Rötungen und vereinzelte petechiale Einblutung keine weiteren Auffälligkeiten [9]. Im Gegensatz hierzu liegen die Komplikationsraten bei der operativen Pseudarthrosenbehandlung deutlich höher. Egol et al. (2012) behandelten 134 Patienten mit Pseudarthrosen der langen Röhrenknochen und konnten zeigen, dass in Abhängigkeit von der notwendigen Anzahl an Revisionsoperationen die Komplikationsrate zwischen 11 und 100\% lag [10]. Moghaddam et al. (2016) operierten 99 Patienten mit atrophen tibialen Pseudarthrosen ein- bzw. zweizeitig und konnten in diesem Kollektiv 34 Komplikationen feststellen (34\%) [11].

Eine konservative Behandlung von Frakturheilungsstörungen erfordert engmaschige klinisch-radiologische Kontrollen, um mögliche oder drohende Komplikationen (Implantatversagen infolge Materialermüdung) rechtzeitig zu erkennen ( $>$ Abb. $\mathbf{3}$ ). Kriterien, die bei der Indikationsstellung zur konservativen Behandlung von Pseudarthrosen erfüllt sein müssen, werden in Liste 1 aufgeführt.

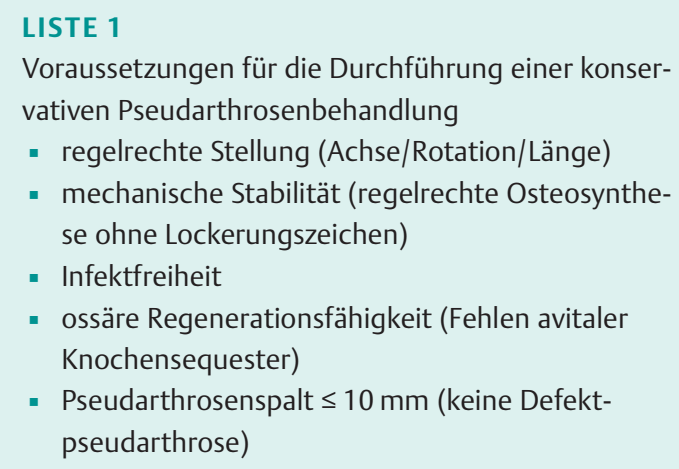

Voraussetzungen für die Durchführung einer konservativen Pseudarthrosenbehandlung

- regelrechte Stellung (Achse/Rotation/Länge)

- mechanische Stabilität (regelrechte Osteosynthese ohne Lockerungszeichen)

- Infektfreiheit

- ossäre Regenerationsfähigkeit (Fehlen avitaler Knochensequester)

- Pseudarthrosenspalt $\leq 10 \mathrm{~mm}$ (keine Defektpseudarthrose)

\section{Belastungssteigerung}

Das „Gesetz zur Transformation der Knochen“ von Julius Wolff und die von Harald M. Frost ergänzte „Mechanostat-Theorie" sind die theoretische Grundlage für die Belastungssteigerung als Möglichkeit einer konservativen Pseudarthrosenbehandlung $[12,13]$. Der entstehende mechanische Reiz wird von Knochenzellen (Osteozyten) registriert, in biochemische Signale innerhalb der Zelle umgewandelt und die Knochenneubildung angeregt (Mechanotransduktion) [14]. 


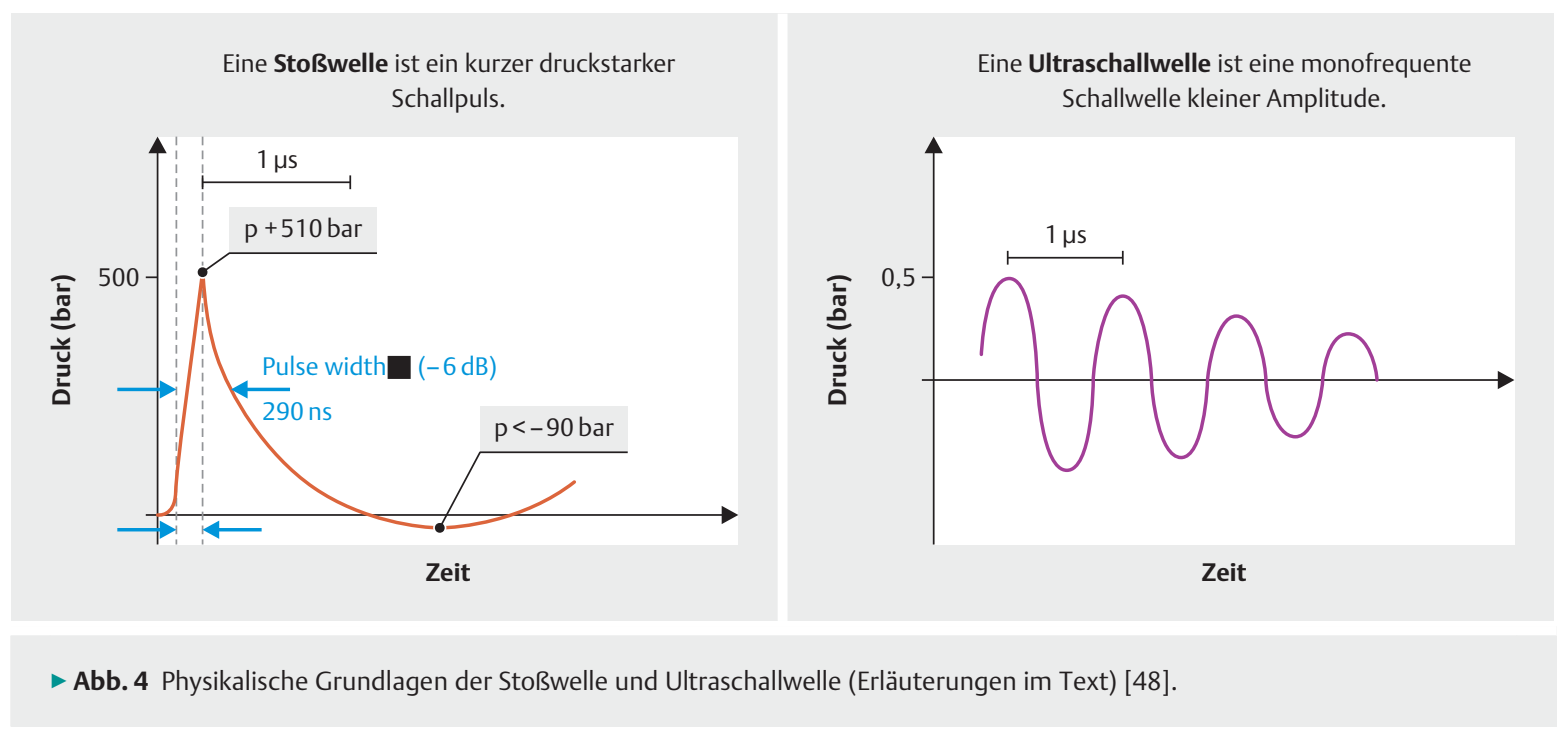

Besonders im Bereich der unteren Extremität kann durch eine Belastungssteigerung eine Stimulierung der Knochenheilung erzielt werden. Houben et al. (2018) konnten in einer kürzlich publizierten Studie die Bedeutung der frühzeitigen Aufbelastung nach operativ behandelter Tibiaschaftfraktur feststellen: Eine längere Entlastungsphase der operierten Extremität resultierte häufiger in eine Frakturheilungsstörung [15]. Sarmiento et al. (2003) berichten über eine konservative Behandlung mittels funktionellem Brace bei verzögerter oder nicht verheilter Tibiaschaftfraktur und symptomorientierter Vollbelastung innerhalb einer Subgruppe (15 Patienten), was zu einer erfolgreichen Konsolidierung geführt hat [16].

Eine gängige Methode bei Pseudarthrosen der unteren Extremität und stabiler osteosynthetischer Versorgung (Liste 1) ist die sukzessive, schmerzadaptierte Aufbelastung um $10 \mathrm{~kg} /$ Woche bis zum Erreichen der Vollbelastung.

\section{Biophysikalische supportive Maßnahmen}

\section{Niedrig intensivierter gepulster Ultraschall (LIPUS)}

Ultraschallwellen sind Signalwellen, die oberhalb des hörbaren menschlichen Frequenzbereichs liegen und sich im Gewebe als sinusförmige Druckwellen ausbreiten. Sie entstehen durch den von Jaques und Pierre Curie im Jahr 1880 entdeckten (indirekten) piezoelektrischen Effekt: Hochfrequente elektrische Spannungen führen zu einer Verformung von Kristallkörpern, sodass sinusförmige Schwingungen entstehen. Ultraschallwellen, die für die Behandlung von Knochenheilungsstörungen eingesetzt werden, besitzen charakteristische physikalische Parameter: Frequenz 1,5 MHz, Repetitionsrate 1 kHz, Impulslänge $200 \mu$ s, Intensität 30 mW/cm² ( Abb. 4).
In zahlreichen grundlagenwissenschaftlichen Studien wurde die Wirkung von LIPUS untersucht. Harrison et al. (2016) konkludieren in ihrer Übersichtsarbeit, dass basierend auf den vorliegenden zellkultur- und tierexperimentellen Studien Nanobewegungen in der Pseudarthrosenregion via Focal Adhesions und Integrinrezeptoren in biochemische Signale umgewandelt werden (Mechanotransduktion) [17]. Die Autoren resümieren, dass eine gesteigerte Expression von COX2 induziert [18] und dadurch die Genexpression weiterer osteogener Faktoren stimuliert wird $[19,20]$. So ergab sich in Untersuchungen von Suzuki et al. (2009) ein Anstieg der BMP-2-, BMP-4und BMP-6-Expression im murinen Frakturmodell nach LIPUS-Behandlung [21].

In der Zwischenzeit liegen zahlreiche klinische Studien zu der Behandlung von Frakturheilungsstörungen mit niedrig intensiviertem gepulstem Ultraschall vor. In der Studie von Nolte et al. (2001) wurden 29 Patienten mit Pseudarthrosen unterschiedlicher Regionen behandelt und eine Konsolidierungsrate von $86 \%$ erzielt [22]. Eine aktuellere Registerstudie von Zura et al. (2015) ermittelte eine identische Heilungsrate von 86,2\% nach LIPUS-Therapie bei 767 Patienten mit chronischen Pseudarthrosen (>365 Tage) [23]. Obwohl dieser Studie eine hohe Fallzahl zugrunde liegt, konnten weitere 519 Patienten u.a. aufgrund Loss-to-Follow-up, Noncompliance und Studienrücktritten trotz Erfüllung der definierten Einschlusskriterien nicht berücksichtigt werden. Bei dem überwiegenden Anteil der 767 eingeschlossenen Patienten (88\%) bestand ein zeitlicher Abstand zwischen LIPUS-Behandlung und letzter Operation von unter 90 Tagen, sodass der Effekt der Ultraschallbehandlung nur schwer eingeschätzt werden kann. Eine deutlich geringere Erfolgsrate als in den vorgenannten Studien ergab sich mit 32,8\% in einer prospektiven Beobachtungsstudie von Biglari et al. (2016) [24]. Die gleiche Arbeitsgruppe analysierte in einer weiteren Studie prospektiv bei 23 Patienten die Se- 
- Tab. 1 Einflussfaktoren auf die Konsolidierungsrate nach LIPUS-Behandlung.

\begin{tabular}{|l|l|l|l|}
\hline \multirow{2}{*}{ Studie } & \multicolumn{2}{|l|}{ Einflussfaktoren auf die Konsolidierungsrate (LIPUS) } \\
\hline & positiv & neutral & negativ \\
\hline Nolte et al., 2001 [22] & & & - Rauchen \\
\hline Jingushi et al., 2007 [49] & $\begin{array}{l}\text { - kurzer Zeitraum Unfall - LIPUS } \\
\text { - kurzer Zeitraum OP - LIPUS }\end{array}$ & $\begin{array}{l}\text { - Alter } \\
\text { - Geschlecht }\end{array}$ & \\
\hline Watanabe et al., 2013 [50] & - hypertrophe Pseudarthrose & - Rauchen & " Marknagelung \\
\hline Biglari et al., 2016 [24] & - Ilattenosteosynthese & " großer Frakturspalt \\
\hline & - Plattenosteosynthese & - hoher NUSS \\
\hline - kurzer Zeitraum Unfall - LIPUS & & " großer Frakturspalt \\
\hline
\end{tabular}

rumkonzentrationen von TGF- $\beta 1$, PDGF und bFGF vor und während der LIPUS-Therapie. 58\% der Patienten $(11 \times)$ zeigten im Verlauf eine knöcherne Heilung der Pseudarthrose nach LIPUS-Behandlung; ein Unterschied in Bezug auf die systemische Zytokinkonzentration konnte gegenüber der Gruppe mit persistierender Pseudarthrose $(8 \times)$ nicht festgestellt werden. Die Ergebnisse von weiteren 4 Patienten wurden aufgrund eines frühzeitigen Behandlungsabbruchs nicht berücksichtigt [25]. Ein signifikanter Unterschied für die Konsolidierungsraten konnte auch in der bislang einzigen randomisierten, placebokontrollierten Studie zwischen der LIPUS-Gruppe und der Placebogruppe nicht nachgewiesen werden (65 vs. 46\%) [26]. Einflussfaktoren, die zu einer höheren Konsolidierungsrate nach LIPUS-Therapie führen, wurden in dieser Arbeit nicht analysiert. Im Gegensatz hierzu existieren zahlreiche weitere Studien, die verschiedene Parameter als Erfolgsvariablen für eine LIPUS-Behandlung für die knöcherne Heilung der Pseudarthrose identifizieren ( $\triangleright$ Tab. 1).

Die Durchführung der Ultraschalltherapie erfolgt in der Häuslichkeit. Der Schallkopf eines batteriebetriebenen Ultraschallgerätes wird unter Verwendung von Kopplungsgel über die Pseudarthrosenregion platziert, die zuvor unter ärztlicher Aufsicht (unter Röntgenkontrolle oder sonografisch) bestimmt und markiert wurde. Hierbei beträgt die Anwendungsdauer $20 \mathrm{~min} /$ Tag über einen Zeitraum von 90-120 Tagen. Eine große Bedeutung kommt entsprechend der Patientencompliance zu: Die tägliche und langandauernde Behandlung (i.d.R. über 90 Tage) erfordert ein hohes Maß an Zuverlässigkeit und Ausdauer seitens der Patienten. Diesbezüglich konnten Raten der Behandlungscompliance zwischen 83 und 91\% gezeigt werden $[25,26]$.

\section{Fokussierte, hochenergetische extrakorporale Stoßwellentherapie (fESWT)}

Bereits nach Ende des Zweiten Weltkriegs fand im militärischen Bereich umfangreiche Forschung zu Stoßwellen statt, sodass Patente für den elektrohydraulischen und elektromagnetischen Stoßwellengenerator Ende der 50er-Jahre erteilt wurden. 20 Jahre später erhielt die Stoßwellentherapie über die Urologie Einzug in die Medizin und wird heute weiterhin erfolgreich zur Lithotripsie angewendet. Erst Anfang der 1990er-Jahre zeigten ersten Studien den erfolgreichen Einsatz der fokussierten extrakorporalen Stoßwellentherapie (fESWT) zur Behandlung der Pseudarthrose zunächst durch Haupt et al. am Tiermodell und kurze Zeit später durch Valchanou und Michailov am Patienten [27, 28].

Die Stoßwelle ist ein mechanisch-akustischer Druckimpuls, gekennzeichnet durch eine hohe Druckamplitude mit schnellem Anstieg gefolgt von einer negativen Druckphase ( $\mathbf{A}$ Abb. 4). Zur Erzeugung der Stoßwellen werden elektrohydraulische, elektromagnetische und piezoelektrische Verfahren unterschieden. Die Welle breitet sich zunächst radial aus und kann zur Behandlung tieferliegender Strukturen mit einem Reflektor fokussiert werden (fESWT). Meist werden die Stoßwellen im Wasser erzeugt, um im biologischen Gewebe ohne signifikante Änderung der Impedanz entkoppelt zu werden. Die Fokuszone wird als der Bereich definiert, in dem die Drücke mehr als die Hälfte des im Zentrum gemessenen Spitzendrucks betragen (Full Width at Half Maximum). Der hierfür angestrebte therapeutische Spitzendruck wird mit > 50 bar definiert. Für die praktische Anwendung haben sich allerdings die applizierte Gesamtenergie (mJ) und die Energieflussdichte $\left(\mathrm{m} / / \mathrm{mm}^{2}\right)$ etabliert. Zur Beschreibung der physikalischen Effekte wird die direkte Wirkung auf akustische Grenzflächen und die durch die DruckZug-Wechselbelastung erzeugte Kavitation und Mikrojets unterschieden [29]. 
Ob die in der Lithotripsie erreichte Zertrümmerungswirkung der Stoßwelle die Knochenheilung durch Mikrofrakturierung anregt, ist heute umstritten. Aktuelle Studien zeigen vielmehr, dass die Stoßwelle über Mechanotransduktion zu einer Expressionssteigerung proangiogenetischer und proosteogenetischer Faktoren führt. Hervorzuheben sind hier die Publikationen der Arbeitsgruppe Wang et al., die nicht nur eine Hochregulation der Transmembranproteinfamilie Integrine zeigen, sondern auch intrazelluläre Signalwegaktivierung, wie z. B. die Phosphorylierung der Focal Adhesion Kinase, p38, MAPK, ERK1/2 und AKT beschreiben. Die anschließende Expression zahlreicher Wachstumsfaktoren und Zytokine (z. B. BMPs und TGF- $\beta$ ) sowie die Differenzierung mesenchymaler Stammzellen zu Osteoprogenitorzellen werden mit Knochenheilung in Zusammenhang gebracht [30,31].

Observationsstudien nach Stoßwellentherapie zeigen unabhängig von Lokalisation und Pseudarthrosentyp Konsolidierungsraten von 60-85\% ( $\mathbf{A b b . 5}$ und 6). Cacchio et al. (2009) konnten in einer randomisiert kontrollierten Level-I-Studie zeigen, dass hypertrophe Pseudarthrosen von Röhrenknochen nach Stoßwellentherapie keine signifikant schlechteren Konsolidierungsraten im Vergleich zur operativen Therapie haben [32]. Hierbei traten in der Stoßwellengruppe keinerlei Komplikationen auf. Furia et al. (2010) konnten ähnliche Ergebnisse bei der Behandlung von Pseudarthrosen der Basis des Mittelfußknochens V im Vergleich zwischen Stoßwelle und Schraubenosteosynthese zeigen [33]. Eine retrospektive Studie zur Skaphoidpseudarthrose ergab gleiche Konsolidierungsraten und Funktionalität beim Vergleich zwischen Stoßwellentherapie und einer operativen Therapie
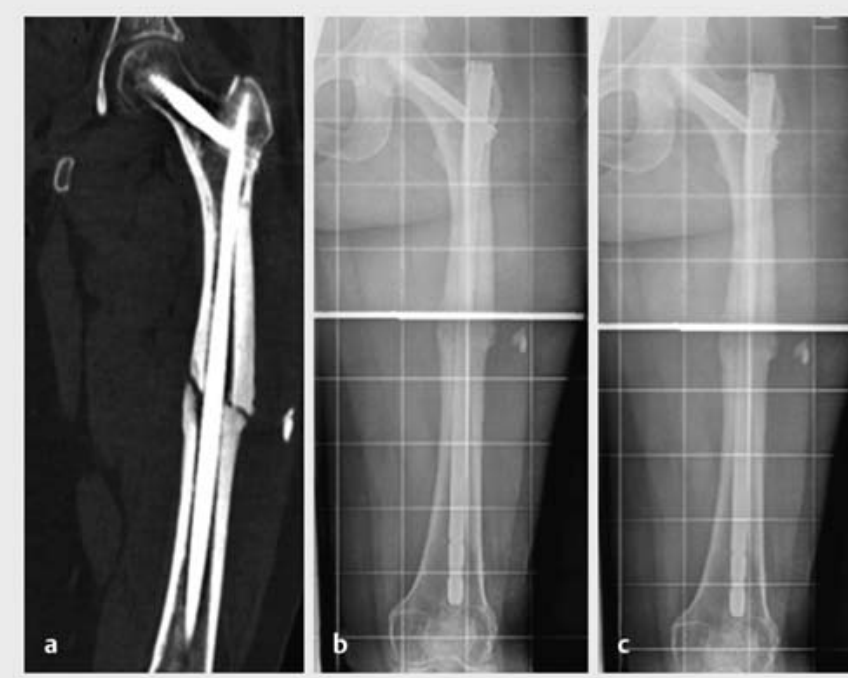

- Abb. 5 a Ausbleibende Frakturheilung einer Femurschaftfraktur, die initial mit einem Marknagel versorgt wurde; 8 Monate vor der Indikationsstellung zur fESWT erfolgte bereits der erfolglose Versuch einer Dynamisierung. b Radiologische Verlaufskontrolle 3 Monate nach fESWT. c Knöcherne Konsolidierung 6 Monate nach fESWT.

in Matti-Russe-Technik [34]. Zahlreiche Level-III-IV-Studien unterstreichen diese Ergebnisse. Insgesamt ist die Stoßwellentherapie bei richtiger Indikationsstellung ein komplikationsarmes Verfahren. Nur selten werden Hautrötungen, Blutergüsse oder Sehnenauflockerungen beschrieben. Im Vergleich zur operativen Therapie können daher nicht nur Komplikationen vermieden, sondern
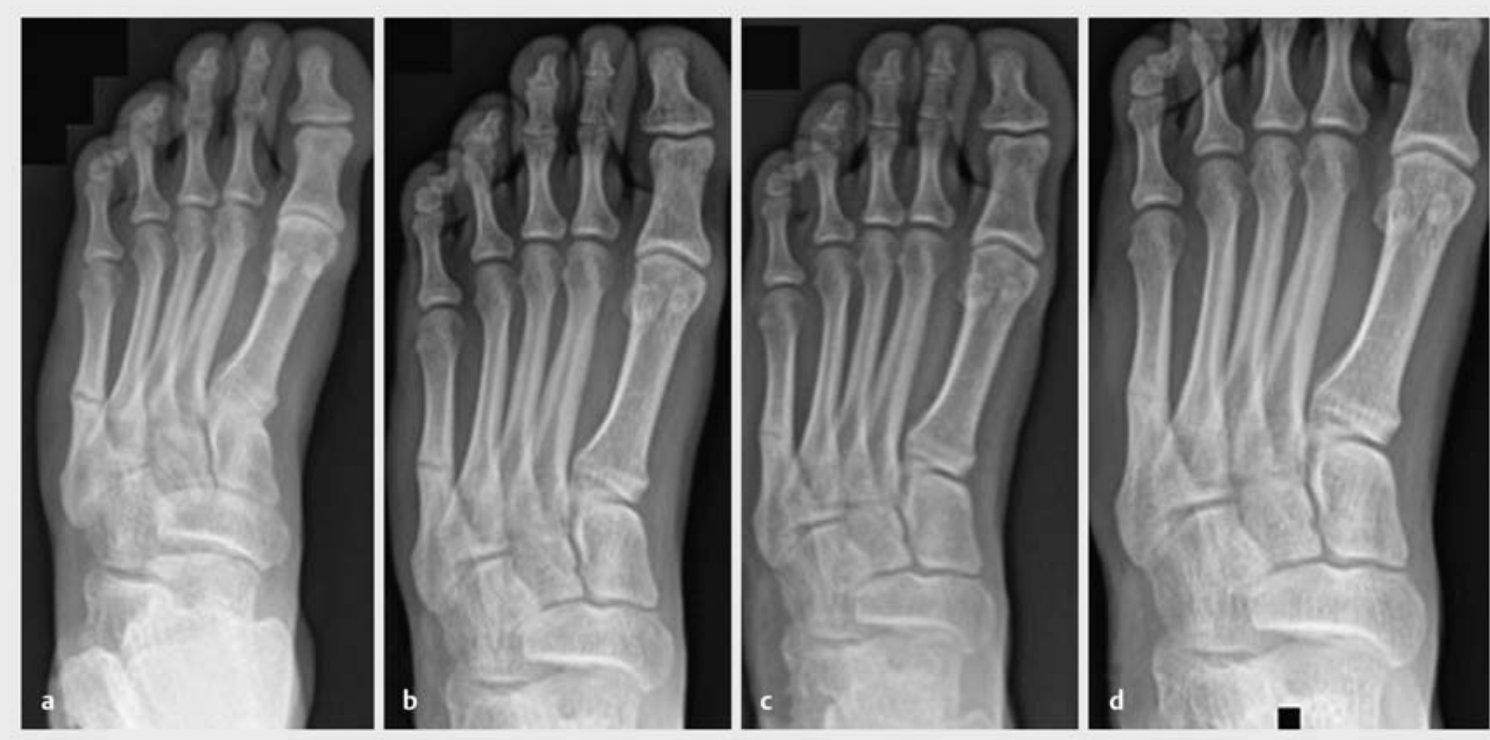

- Abb. 6 a Pseudarthrose im Bereich des diaphysären 5. Mittelfußknochens. b-d Radiologische Verlaufskontrolle 6 Wochen, 3 Monate und 6 Monate nach fESWT mit abschließendem Nachweis der Konsolidierung. 
\Tab.2 Möglichkeiten der elektrischen und elektromagnetischen Therapie mit Vor- und Nachteilen. Abkürzungen siehe Text.

\begin{tabular}{|c|c|c|c|}
\hline Verfahren & Methode & Vorteile & Nachteile \\
\hline DC & $\begin{array}{l}\text { operatives Einbringen einer Kathode } \\
\text { frakturnah und einer Anode subkutan } \\
\text { (direkte Stromapplikation) }\end{array}$ & $\begin{array}{l}\text { - konstanter Reiz } \\
\text { - Compliance } \uparrow \\
\text { - gute Position der Elekt- } \\
\text { rode }\end{array}$ & $\begin{array}{l}\text { - Operation notwendig } \\
\text { - OP-Komplikationen: Infekt, Dislokation der } \\
\text { Elektrode, Schmerzen durch Implantat } \\
\text { - Implantatentfernung erforderlich (OP) }\end{array}$ \\
\hline PEMF & $\begin{array}{l}\text { Positionierung einer Magnetfeldspule } \\
\text { über der Frakturregion, Induktion eines } \\
\text { Magnetfeldes (induktives Verfahren) }\end{array}$ & - nicht invasiv & $\begin{array}{l}\text { - dosisabhängige Wirkung } \\
\text { - Therapie 3-10 h/d } \\
\text { - Compliance } \downarrow\end{array}$ \\
\hline CC & $\begin{array}{l}\text { Induktion eines elektrischen Feldes } \\
\text { durch oszillierenden Strom zwischen } \\
2 \text { Klebeelektroden (perkutan) }\end{array}$ & - nicht invasiv & $\begin{array}{l}\text { - Therapie } 24 \mathrm{~h} / \mathrm{d} \\
\text { - Compliance } \downarrow \\
\text { - Hautirritation } \\
\text { - Eindringtiefe begrenzt }\end{array}$ \\
\hline CMF & $\begin{array}{l}\text { konstante sinusförmige elektrische } \\
\text { Stimulierung durch } 2 \text { externe Spulen } \\
\text { über der Frakturregion }\end{array}$ & $\begin{array}{l}\text { - } \text { nicht invasiv } \\
\text { - } 30 \text { min } / d \\
\text { - Compliance } \uparrow\end{array}$ & \\
\hline
\end{tabular}

auch mehr als das 4-Fache der Behandlungskosten eingespart werden $[35,36]$.

Die Stoßwellentherapie wird ambulant entsprechend den DIGEST-Leitlinien (DIGEST = Deutschsprachige Internationale Gesellschaft für Extrakorporale Stoßwellentherapie) durchgeführt. In Abhängigkeit von der Stoßwellengenerierung erfolgen 3-4 Sitzungen in einem Intervall von 3-7 Tagen (elektromagnetische Stoßwelle) oder eine einmalige Sitzung bei Behandlung mit einem elektrohydraulischen Stoßwellengerät (Wiederholung nach 3-6 Monaten möglich). In letzterem Fall ist eine Narkose notwendig. Im eigenen Vorgehen wird aufgrund der hohen Patientencompliance die fESWT gegenüber den weiteren biophysikalischen Verfahren favorisiert.

\section{Elektrische und elektromagnetische Therapie}

Die mechanische Belastung des Knochens führt zu einer Knochenneubildung (siehe Abschnitt Belastungssteigerung) $[12,13]$. Ein Erklärungsmodell hierfür wurde u.a. durch Yasuda (1977) beschrieben: Elektronegative Ladungen treten in einer druckbelasteten knöchernen Region auf und führen zu einer Kallusbildung, elektropositive Potenziale werden in dem zugbelasteten knöchernen Bereich registriert und resultieren in einem Knochenabbau [37]. Diese Erkenntnis wurde als Grundlage für die elektrische und elektromagnetische Behandlung von Frakturheilungsstörungen herangezogen. In der klinischen Anwendung finden sich 4 unterschiedliche Verfahren: Direct Current (DC), Pulsed electromagnetic Field (PEMF), Capacitive Coupling (CC) und Combined magnetic Fields (CMFs), von denen die pulsierenden elektromagnetischen Felder (PEMF) am häufigsten eingesetzt werden ( $\vee$ Tab. 2). Die Stimulationsparameter der unterschiedlichen auf dem Markt befindlichen PEMF-Geräte weisen dabei eine hohe Variabilität in Bezug auf Frequenz, Stromstärke und maximale Magnetfelddichte auf
[38]. Behandlungszeiten variieren zwischen 3 und 10 Stunden am Tag [39-41].

Die zelluläre und molekulare Wirkung von PEMF wurde in der Vergangenheit in verschiedenen Studien analysiert. Ongaro et al. (2014) konnten zeigen, dass die osteogene Differenzierung von humanen mesenchymalen Stammzellen (hMSC) unterschiedlicher Herkunft (Knochenmark, Fettgewebe) durch PEMF induziert wird [42]. Schwartz et al. (2008) untersuchten in einer Zellkulturstudie den osteogenen Effekt von BMP-2 nach PMEF-Therapie auf hMSC und konnten einen Vorteil gegenüber den weiteren Interventions- bzw. Kontrollgruppen zeigen [43]. Trotz der zahlreichen experimentellen Untersuchungen zur biologischen Antwort des knöchernen Gewebes auf die PEMF-Behandlung bleibt der genaue Wirkmechanismus unklar. Zuletzt wurde in Zellkulturstudien dem MAP-Kinase- (ERK 1/ERK 2) und NOTCH-Signalweg eine bedeutende Rolle zugeschrieben $[44,45]$.

Die PEMF-Therapie ist seit 1979 durch die Food and Drug Administration (FDA) als additives Verfahren zur Unterstützung der Knochenheilung bei Pseudarthrosen und Arthrodesen zugelassen. In einer randomisiert kontrollierten, doppelblinden Studie ergab sich für die PEMF-Behandlung von nicht verheilten Frakturen langer Röhrenknochen (Frakturalter 4-6 Monate) eine Konsolidierungsrate von 38,7 gegenüber 22,2\% (PEMF-Gruppe vs. Kontrollgruppe) nach 3 Monaten (nicht signifikant) [40]. Assiotis et al. (2012) behandelten in einer prospektiven Beobachtungsstudie 44 Patienten mit tibialen Frakturheilungsstörungen und konnten nach PEMF-Therapie eine Heilungsrate von 77,3\% feststellen. Einen Zusammenhang der Konsolidierungswahrscheinlichkeit mit Alter, Frakturseite (rechts vs. links), Frakturtyp (einfach vs. mehrfragmentär und offen vs. geschlossen), Rauchverhalten, Diabetes oder Osteosyntheseart (Platten- vs. Marknagelosteosynthese) konnte nicht nachgewiesen 
werden. Allerdings berichten die Autoren über eine tendenziell höhere Heilungsrate mit zunehmender Anwendungsdauer [39]. Ebenso zeigten Murray und Pethica (2016) eine Verkürzung der Konsolidierungszeit der Fraktur infolge längerer täglicher PEMF-Behandlung [41]. In weiteren Studien konnten Auswirkungen auf die lokale Expression von Wachstumsfaktoren durch pulsierende elektromagnetische Felder nur sehr eingeschränkt bestätigt werden. Während für Placental Growth Factor (PIGF) ein Anstieg verzeichnet werden konnte, traf dies für alle weiteren analysierten Wachstumsfaktoren (u.a. BMP-5 und BMP-7) nicht zu [46].

Die Behandlung mit pulsierenden elektromagnetischen Feldern kann ambulant durchgeführt werden. Die Magnetfeldspule wird hierzu über die Frakturregion positioniert. Die tägliche Behandlungsdauer (mehrstündig) über einen mehrwöchigen Zeitraum variiert in Abhängigkeit vom verwendeten Gerät entsprechend den Herstellerempfehlungen. Ebenso unterscheiden sich die physikalischen Einstellungsparameter der Geräte. Ähnlich wie bei der Ultraschallbehandlung ist eine hohe Patientencompliance erforderlich.

\section{Biophysikalische Verfahren: sinnvoll oder nicht?}

Der Sinn einer Stimulierung der Frakturheilung durch biophysikalische Verfahren wird häufig diskutiert. Anders als die Modifikation der zellulären Aktivität durch chemische bzw. synthetische Agenzien (Medikamente, Wachstumsfaktoren) ist die Möglichkeit einer physikalischen Beeinflussung des zellulären Metabolismus in der Frakturheilung kontrovers. Trotz zahlreicher grundlagenwissenschaftlicher Studien, in denen eine Wirkung der verschiedenen biophysikalischen Verfahren auf den Knochenmetabolismus festgestellt wurde, steht der sichere Nachweis einer klinischen Relevanz dieser Verfahren anhand guter LevelI-Studien weiter aus. Zwar zeigen die aktuell in der Literatur zahlreich vorhandenen Level-III-IV-Studien überwiegend einen positiven Effekt in der Behandlung von Pseudarthrosen, diese sind aber aufgrund ihres Studiendesigns nur sehr eingeschränkt zu verwerten. Darüber hinaus ist ein Vergleich der Studien untereinander infolge der Vielzahl an verschiedenen physikalischen Stimulierungsparametern häufig nicht möglich. Hier bedarf es zukünftig weiterer hochwertiger randomisierter, kontrollierter Studien (RCT) wie von Cacchio et al. (2009) zur fESWT oder Schofer et al. (2010) zur Behandlung von Pseudarthrosen mittels Ultraschall $[26,47]$. Hinsichtlich der Wirksamkeit von PEMF ist aktuell durch den Gemeinsamen Bundesausschuss ein Beratungsverfahren zu einer Richtlinie zur Erprobung dieser Therapie eingeleitet. Hier bleibt abzuwarten, ob auf Basis einer in diesem Zusammenhang möglicherweise initiierten RCT neue Erkenntnisse gewonnen werden.

\section{Fazit für die Praxis}

Die konservative Therapie einer verzögerten Frakturheilung oder Pseudarthrose ist gerade in frühen Phasen eine effiziente Therapiealternative. Hierbei kann unter strenger Indikationsstellung die Frakturheilung durch Belastungssteigerung oder biophysikalische Verfahren (LIPUS, fESWT, PEMF) angeregt werden. Letztere sind im Vergleich zu einer Revisionsoperation besonders aufgrund geringerer Komplikationen und Kosten sehr attraktiv. Im eigenen Vorgehen wird die fESWT favorisiert und mit gutem Erfolg eingesetzt.

\section{Interessenkonflikt}

Die Autoren geben an, dass kein Interessenkonflikt besteht.

Autoren
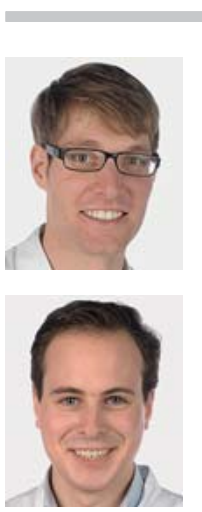

\section{J. Everding}

Dr. med., Facharzt, Klinik für Unfall-, Handund Wiederherstellungschirurgie, Universitätsklinikum Münster

\section{J. Stolberg-Stolberg}

Dr. med., Wissenschaftlicher Mitarbeiter, Klinik für Unfall-, Hand- und Wiederherstellungschirurgie, Universitätsklinikum Münster

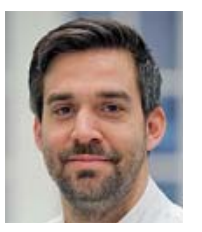

\section{S. Roßlenbroich}

Dr. med., Oberarzt, Klinik für Unfall-, Handund Wiederherstellungschirurgie, Universitätsklinikum Münster

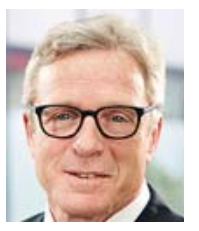

\section{Michael J. Raschke}

Univ.-Prof. Dr. med., Direktor, Klinik für Unfall-, Hand- und Wiederherstellungschirurgie, Universitätsklinikum Münster

Korrespondenzadresse

Dr. med. Jens Everding

Klinik für Unfall-, Hand- und Wiederherstellungschirurgie Universitätsklinikum Münster

Waldeyer Straße 1

48149 Münster

Tel.: 0251/83-56301

Fax: 0251/83-56318

jens.everding@ukmuenster.de 
Literatur

[1] Tzioupis C, Giannoudis PV. Prevalence of long-bone nonunions. Injury 2007; 38 (Suppl. 2): S3-S9. doi:10.1016/ S0020-1383(07)80003-9

[2] Zura R, Xiong Z, Einhorn T et al. Epidemiology of fracture nonunion in 18 human bones. JAMA Surg 2016; 151: e162775. doi:10.1001/jamasurg.2016.2775

[3] Calori GM, Albisetti W, Agus A et al. Risk factors contributing to fracture non-unions. Injury 2007; 38 (Suppl. 2): S11-S18. doi:10.1016/50020-1383(07)80004-0

[4] Weber BG, Czech O. Pseudarthrosen - Pathophysiologie, Biomechanik, Therapieergebnisse. Bern, Stuttgart, Wien: Huber; 1973

[5] Calori GM, Phillips M, Jeetle S et al. Classification of non-union: Need for a new scoring system? Injury 2008; 39 (Suppl. 2): S59-S63. doi:10.1016/S0020-1383(08)70016-0

[6] Giannoudis PV, Einhorn TA, Marsh D. Fracture healing: the diamond concept. Injury 2007; 38 (Suppl. 4): S3-S6

[7] Higgins A, Glover M, Yang Y et al. EXOGEN ultrasound bone healing system for long bone fractures with non-union or delayed healing: A NICE medical technology guidance. Appl Health Econ Health Policy 2014; 12: 477-484. doi:10.1007| s40258-014-0117-6

[8] Furia JP, Juliano PJ, Wade AM et al. Shock wave therapy compared with intramedullary screw fixation for nonunion of proximal fifth metatarsal metaphyseal-diaphyseal fractures. J Bone Joint Surg Am 2010; 92: 846-854. doi:10.2106/JBJS.I.00653

[9] Everding ], Freistuhler M, Stolberg-Stolberg] et al. [Extracorporal shock wave therapy for the treatment of pseudarthrosis: new experiences with an old technology]. Unfallchirurg 2017; 120: 969-978. doi:10.1007/s00113-016-0238-5

[10] Egol KA, Bechtel C, Spitzer AB et al. Treatment of long bone nonunions: factors affecting healing. Bull NYU Hosp Jt Dis 2012; 70: 224-231

[11] Moghaddam A, Thaler B, Bruckner T et al. Treatment of atrophic femoral non-unions according to the diamond concept: Results of one- and two-step surgical procedure. J Orthop 2016; 14: 123-133. doi:10.1016/j.jor.2016.10.003

[12] Wolff J. Das Gesetz der Transformation der Knochen. Berlin: A. Hirschwald; 1892

[13] Frost HM. Skeletal structural adaptations to mechanical usage (SATMU): 1. Redefining Wolff's law: The bone modeling problem. Anat Rec 1990; 226: 403-413. doi:10.1002/ ar.1092260402

[14] Haffner-Luntzer M, Liedert A, Ignatius A. [Mechanobiology and bone metabolism: clinical relevance for fracture treatment]. Unfallchirurg 2015; 118: 1000-1006. doi:10.1007/ s00113-015-0102-z

[15] Houben IB, Raaben M, Van Basten Batenburg M et al. Delay in weight bearing in surgically treated tibial shaft fractures is associated with impaired healing: a cohort analysis of 166 tibial fractures. Eur J Orthop Surg Traumatol 2018; 28: 1429-1436. doi:10.1007/s00590-018-2190-2

[16] Sarmiento A, Burkhalter WE, Latta LL. Functional bracing in the treatment of delayed union and nonunion of the tibia. Int Orthop 2003; 27: 26-29. doi:10.1007/s00264-002-0405-x

[17] Harrison A, Lin S, Pounder $N$ et al. Mode \& mechanism of low intensity pulsed ultrasound (LIPUS) in fracture repair. Ultrasonics 2016; 70: 45-52. doi:10.1016/j.ultras.2016.03.016

[18] Reher P, Harris M, Whiteman M et al. Ultrasound stimulates nitric oxide and prostaglandin E2 production by human osteo- blasts. Bone 2002; 31: 236-241. doi:10.1016/S8756-3282 (02)00789-5

[19] Leung KS, Cheung WH, Zhang C et al. Low intensity pulsed ultrasound stimulates osteogenic activity of human periosteal cells. Clin Orthop Relat Res 2004; (418): 253-259

[20] Hasegawa T, Miwa M, Sakai Y et al. Osteogenic activity of human fracture haematoma-derived progenitor cells is stimulated by low-intensity pulsed ultrasound in vitro. J Bone Joint Surg $\mathrm{Br}$ 2009; 91: 264-270. doi:10.1302/0301-620X.91B2.20827

[21] Suzuki A, Takayama T, Suzuki N et al. Daily low-intensity pulsed ultrasound stimulates production of bone morphogenetic protein in ROS17/2.8 cells. J Oral Sci 2009; 51: 2936. doi:10.2334/josnusd.51.29

[22] Nolte PA, van der Krans A, Patka P et al. Low-intensity pulsed ultrasound in the treatment of nonunions. J Trauma 2001; 51: 693-702

[23] Zura R, Della Rocca G], Mehta S et al. Treatment of chronic ( $>1$ year) fracture nonunion: Heal rate in a cohort of 767 patients treated with low-intensity pulsed ultrasound (LIPUS). Injury 2015; 46: 2036-2041. doi:10.1016/j.injury.2015.05.042

[24] Biglari B, Yildirim TM, Swing T et al. Failed treatment of long bone nonunions with low intensity pulsed ultrasound. Arch Orthop Trauma Surg 2016; 136: 1121-1134. doi:10.1007| s00402-016-2501-1

[25] Moghaddam A, Yildirim TM, Westhauser F et al. Low intensity pulsed ultrasound in the treatment of long bone nonunions: evaluation of cytokine expression as a tool for objectifying nonunion therapy. J Orthop 2016; 13: 306-312. doi:10.1016/ j.jor.2016.06.028

[26] Schofer MD, Block JE, Aigner J et al. Improved healing response in delayed unions of the tibia with low-intensity pulsed ultrasound: results of a randomized sham-controlled trial. BMC Musculoskelet Disord 2010; 11: 229. doi:10.1186/14712474-11-229

[27] Vincent KCS, d'Agostino MC. History of Shock Wave Treatment and its basic Principles. In: Wang C-J, Schaden W, Ko JY, eds. Shockwave Medicine. Vol 6. Basel: Karger; 2018: 1-16

[28] Shrivastava SK, Kailash. Shock wave treatment in medicine. J Biosci 2005; 30: 269-275

[29] Wess O. Physikalische Grundlagen der extrakorporalen Stoßwellentherapie. J Min Stoffwechs 2004; 11: 7-18

[30] Wang C]. Extracorporeal shockwave therapy in musculoskeletal disorders. J Orthop Surg Res 2012; 7: 11. doi:10.1186/ 1749-799X-7-11

[31] Schaden W, Mittermayr R, Haffner $N$ et al. Extracorporeal shockwave therapy (ESWT)-first choice treatment of fracture non-unions? Int J Surg 2015; 24(Pt B): 179-183. doi:10.1016/ j.jjsu.2015.10.003

[32] Cacchio A, Giordano L, Colafarina O et al. Extracorporeal shock-wave therapy compared with surgery for hypertrophic long-bone nonunions. J Bone Joint Surg Am 2009; 91: 25892597. doi:10.2106/JBJS.H.00841

[33] Furia JP, Juliano PJ, Wade AM et al. Shock wave therapy compared with intramedullary screw fixation for nonunion of proximal fifth metatarsal metaphyseal-diaphyseal fractures. J Bone Joint Surg Am 2010; 92: 846-854. doi:10.2106/JBJS.I.00653

[34] Notarnicola A, Moretti L, Tafuri S et al. Extracorporeal shockwaves versus surgery in the treatment of pseudoarthrosis of the carpal scaphoid. Ultrasound Med Biol 2010; 36: 13061313. doi:10.1016/j.ultrasmedbio.2010.05.004

[35] Moya D, Ramon S, Schaden W et al. The role of extracorporeal shockwave treatment in musculoskeletal disorders. J Bone Joint Surg Am 2018; 100: 251-263. doi:10.2106/JBJS.17.00661 
[36] Everding J, Roßlenbroich S, Raschke MJ. Ultraschall und Stoßwelle in der Pseudarthrosentherapie. Trauma Berufskrankh 2017; 19 (Suppl. 3): S260-S266. doi:10.1007/s10039017-0310-6

[37] Yasuda I. Electrical callus and callus formation by electret. Clin Orthop Relat Res 1977; (124): 53-56

[38] Schmidt-Rohlfing B, Silny ], Niethard FU. [Pulsating electromagnetic fields in treatment of injuries and illnesses of the locomotor system-an overview and meta-analysis]. Z Orthop Ihre Grenzgeb 2000; 138: 379-389. doi:10.1055/s-200010165

[39] Assiotis A, Sachinis NP, Chalidis BE. Pulsed electromagnetic fields for the treatment of tibial delayed unions and nonunions. A prospective clinical study and review of the literature. J Orthop Surg Res 2012; 7: 24. doi:10.1186/1749-799X7-24

[40] Shi HF, Xiong J, Chen YX et al. Early application of pulsed electromagnetic field in the treatment of postoperative delayed union of long-bone fractures: a prospective randomized controlled study. BMC Musculoskelet Disord 2013; 14: 35. doi:10.1186/1471-2474-14-35

[41] Murray HB, Pethica BA. A follow-up study of the in-practice results of pulsed electromagnetic field therapy in the management of nonunion fractures. Orthop Res Rev 2016; 8: 67-72. doi:10.2147/ORR.S113756

[42] Ongaro A, Pellati A, Bagheri L et al. Pulsed electromagnetic fields stimulate osteogenic differentiation in human bone marrow and adipose tissue derived mesenchymal stem cells. Bioelectromagnetics 2014; 35: 426-436. doi:10.1002/ bem. 21862

[43] Schwartz Z, Simon B], Duran MA et al. Pulsed electromagnetic fields enhance BMP-2 dependent osteoblastic differentiation of human mesenchymal stem cells. J Orthop Res 2008; 26: 1250-1255. doi:10.1002/jor.20591

[44] Yong Y, Ming ZD, Feng L et al. Electromagnetic fields promote osteogenesis of rat mesenchymal stem cells through the PKA and ERK1/2 pathways. J Tissue Eng Regen Med 2016; 10: E537-E545. doi:10.1002/term.1864

[45] Bagheri L, Pellati A, Rizzo P et al. Notch pathway is active during osteogenic differentiation of human bone marrow mesenchymal stem cells induced by pulsed electromagnetic fields. J Tissue Eng Regen Med 2018; 12: 304-315. doi:10.1002/ term. 2455

[46] Streit A, Watson BC, Granata JD et al. Effect on clinical outcome and growth factor synthesis with adjunctive use of pulsed electromagnetic fields for fifth metatarsal nonunion fracture: a double-blind randomized study. Foot Ankle Int 2016; 37: 919-923. doi:10.1177/1071100716652621

[47] Cacchio A, Giordano L, Colafarina O et al. Extracorporeal shock-wave therapy compared with surgery for hypertrophic long-bone nonunions. J Bone Joint Surg Am 2009; 91: 25892597. doi:10.2106/JBJS.H.00841

[48] Steinacker T, Steuer M. Use of extracorporeal shockwave therapy (ESWT) in sports orthopedics. Sportverletz Sportschaden 2001; 15: 45-49. doi:10.1055/s-2001-14817

[49] Jingushi S, Mizuno K, Matsushita T et al. Low-intensity pulsed ultrasound treatment for postoperative delayed union or nonunion of long bone fractures. J Orthop Sci 2007; 12: 35-41. doi:10.1007/s00776-006-1080-3

[50] Watanabe Y, Arai Y, Takenaka N et al. Three key factors affecting treatment results of low-intensity pulsed ultrasound for delayed unions and nonunions: Instability, gap size, and atrophic nonunion. J Orthop Sci 2013; 18: 803-810. doi:10.1007/s00776-013-0415-0

\section{Bibliografie}

DOI https://doi.org/10.1055/a-0899-0068

OP-JOURNAL 2019; 35: 252-261 @ Georg Thieme Verlag KG Stuttgart · New York ISSN 0178-1715 